\title{
Determination of Color and Shape Features Based Image Retrieval with Hybrid Feature Descriptor Construction (HFDC)
}

\author{
Aranga Arivarasan* and M. Karthikeyan \\ Division of Computer and Information Science, Annamalai University, Chidambaram, \\ Tamil Nadu, India; profarivarasan@yahoo.co.in, mkshkarthik@yahoo.co.in
}

\begin{abstract}
Objectives: To implement a hybrid feature descriptor construction (HFDC) model to exercise the CBIR. Method/analysis: The model that combines color and shape features of visual content to produce a feature level fusion scheme is introduced. The RGB histogram, HSV histogram and the canny edge histogram features are extracted and fused to produce a hybrid feature vector. Then the determined feature vector through the fusion of the entire dataset is used to train the SVM using RBF kernel to retrieve relevant visual content through identifying the color distribution, and shape is focused here as the main objective. Since from the very beginning of the data usage to surf web information the classification of the similar related objects has been potentially provided a helpful contribution towards helping the users to identify and determine required knowledge from the large corpus of available digital information. Many algorithms and improvements have been in implementation, but the large quantity of available information provides complexity to these techniques to enhance computational hike. This feature level fusion contributes to reducing the overhead. Finding: The HFDC evolutions significantly contribute to achieving an accuracy of $84.60 \%$. The experimental results have proven the efficiency of the HFDC by providing the maximum classification accuracy. Novelty: The results evidently show that (1) HDFC improve the performance by enhancing the feature level fusion process, (2) the fusion procedure produces increased solid and high indicative rendering and (3) by performing feature level fusion of data a core dictionary is provided for better CBIR performance. Improvement: In addition to color and shape feature fusion the texture features can also be combined to improve the performance significantly.
\end{abstract}

Keywords: CBIR, HFDC, RGB, HSV, Canny

\section{Introduction}

The awareness in the prospective usage of visual data has amplified extremely in a short span of time with the rapid growth of the World-Wide Web. With the increased ability of the usage of digital information through a distributed environment many professional users tend to uses this environment to explore their professional skills. This also leads to achieve their goals as well as gather their field of knowledge through remotely stored visual information. But as a process, this is not easy. The users spend a lot of time and energy in the discovery of the process to locate the most wanted image in a huge and diverse compilation of data corpus which leads to considerable irritation. To facilitate the extraction of suitable features to enhance the CBIR, a variety of feature extraction and collection algorithms are analyzed and the efficiency is calculated. 1 In search of the solution for these problems reveled around the image retrieval is widely understood through the increased interest with active research and development area. Convenient approaches and a wide span of promising application for CBIR expertise has been identified in image mining: (a) Crime Prevention, $\underline{\underline{2}}$ (b) The military, (c) academic property, (d) Architectural engineering design, (e) Fashion interior design, (f) Journalism and publicity, (g) Medical diagnosis,- (h) Environmental information

*Author for correspondence 
remote sense systems,,$\underline{4}$, (i) Cultural tradition, ( $\mathrm{j}$ ) Education and training, (k) Home entertainment and $(\mathrm{k})$ Web applications. Crime hindrance agency normally has to maintain huge collections of visual proof counting past suspects' facial photographs fingerprints shoeprints and so on to reduce criminal activities. Typical areas involving CBIR are as follows. In the development of Art galleries and museum management, the CBIR plays an essential role. This model mainly aims to observe how the past social and cultural aspects like learning, social division, profession, politics and income might manipulate an influence towards the current society. ${ }^{-}$In Architecture for engineering design CBIR system, various types of visual evidence are being referred day to day. Basically, the word "architecture" usually refers to building design and production. In engineering, engineers tend to use the production of designing processes to solve the difficulty in the development of a new product. In these areas, the CBIR system plays a significant responsibility to satisfy the user requirement. Interior design is one of the areas which are closely related to building architecture. Interior design has come up to exploit because of the increased possibility and requirements of interior designers in recent times due to improve the design technology and also to improve the interior design of a building. Structural technological elements of buildings that have to be constructed are to be visualized in a beautiful manner. In the area of remote sensing for managing earth assets the agricultural, commodities, water management, soil governing, forestry, weather, snowpack and so on are considered. In the retailing area, the online approach of the retail gap continues to explore because of the current clients progressively utilizing a more convenient remote buying experience. To provide this expertise the CBIR plays a major role. To impose Law and criminal 2 examination the documents like passports, credit cards, laptops, or mobile phones are helpful through the computerized method to identify people through their biometric features of modern lifestyle. Biometric recognition refers to the automatic detection of persons depending on their behavioral characteristics. Web image searching provides the reduced gap between identifying images on the Web and the necessity of image search engines with increased accuracy. There is always a requirement for software to avoiding access to pornographic images. Text-based search engines have developed in a hurry as the Web has expanded its usage widely. Many of the above application areas are in need of successful sensing of both text and images on the Www because of the development in information retrieval in the last five years as a vital foundation of knowledge and entertainment.

In the field of Education and training new skill is introduced through the way user create access and use information. The design and development of digital libraries depend on the computer, communication and other technical skills. The global digital atmosphere can only be shaped while enough active participants have the motivation to design, construct and manage active digital libraries. Universal access to digital libraries and information services are important goals. Digital library collections are not limited to document as a substitute, but they also include digital experiment investigation that cannot be represented or distributed in printed formats. In a broad sense digital library is an automated system that allows users to obtain a consistent means of access to an organized, digital stored repository of information and data. The digital library is an electronic document collection consisting of information in the digital form analog and digitized form various mediums.

Chapter 2 consists of materials and methods involved in the study. In chapter 3 the experimental results were discussed in detail. Chapter 4 describes the conclusion of the proposed method. Finally, chapter 5 discusses the reference made for the proposed work in detail.

\section{Materials and Methods}

\subsection{Color Quantization}

Color quantization ${ }^{7}$ is performed where it is essential to reduce the color information of an image. One of the universal situations is to modify a 24-bit color image into an 8-bit color image. It is also in functional with the intention of generating the color histograms, through minimizing the number of colors utilized to characterize an image. Two conclusions contain to be identified, one is that which are the colors of the superior color set continue to be present in the newly obtained image, and the second one is that the way through which redundant colors are represented to the surplus ones. The fundamental approach to represent an eight-bit image from a 24-bit image is by allocating three bits to the two colors red and green and, because of the human eye's minimal understanding of the blue color, it is assigned two bits. This consideration facilitates to exhibit eight various tones for red and green and four for the blue. This 
procedure will lead to poor outcomes because an image capably can provide various blue tones. At this point all blue tones are grouped just about an assured significance value in an attempt to make merely one tone of blue is considered in an eight-bit image and the leftover three blue tones are never considered. The quantization proposal is resolved through a color space and performing a separation of the color space. While performing a regular quantization method on a determined color space, every axis is separated to a proportion of separation, the axes are separated to $\mathrm{k}, \mathrm{l}$, and $\mathrm{m}$ partitions, and the number of colors $\mathrm{n}$ is derived to symbolize an image will be $\mathrm{n}=\mathrm{k} .1 \mathrm{~m}$, which is frequently specified as an n-bins quantization scheme. The separation of available axis relies on the color space which is to be considered to chosen.

\subsection{RGB Histogram}

The capability of understanding the histograms will provide a suitable understanding of correct knowledge about the image. The histogram $\underline{\underline{8}}$ of an image is obtained through a bar diagram while the horizontal axis representing the tonal range of your image. In an image, each pixel contains a mathematical tonal value representing the primary color. The vertical axis represents the comparative value of pixels ranging from zero through 255. The RGB histogram is used to visualize the histogram of all entities of color channel red color, green color, and blue available in an image as a pixel. One of the setbacks in the regular histogram is that it attempts to dumps all color channels simultaneously. It makes the user hard to identify a specific color channel. Alternatively, the RGB histogram is identical towards regular histogram allowing the user to have individual histograms for all three-color channels. In RGB histogram all color channel histograms will be significantly diverse. It is heavily dependent on the colors which the image contains.

\subsection{HSV Histogram}

HSV is a general color model that is over and over again used instead of the RGB sculpts. In addition, HSV may be identified HSB (hue, saturation and brightness). $?$ The RGB and CMYK are very much related to the primary colors, but the HSV is defined as similar to how humans understand color. This color sculpts describe its colors known as hue or tint as their shadow which is represented as the total quantity of gray value as their brightness for value. Simultaneously the HSB, which

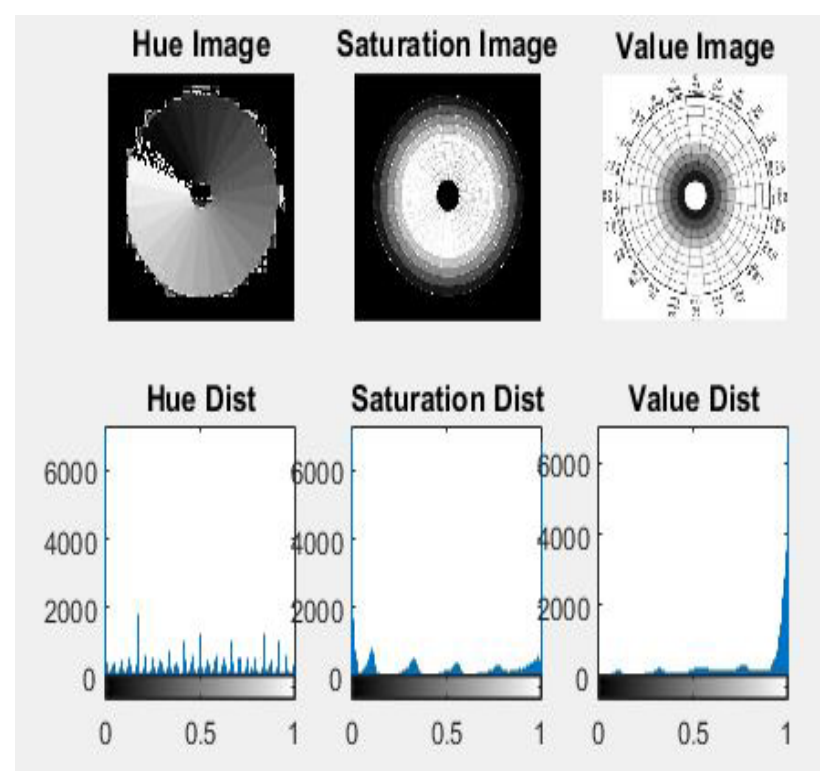

Figure 1. HSV color descriptor.

replaces the term brightness for value refers to the same color model. It is much easy to work with HSV because to isolate the color present in an image is an easier task. So, it is necessary to convert the image to HSV. The HSV color represents the hue cause to occur the color you want, saturation brings how intense the color is in the image and value defines the lightness of the image in Figure 1. The wheel denotes a mild red color and 240 denote a blue color. In MATLAB, the hue ranges from 0 to 1 instead of 0 to 360 . The HSV color circle is described as either a cone shape or cylinder shape with these three apparatuses. Hue is the color segment of the color model, which is expressed in terms of numeral from 0 to 360 degrees. Saturation is the total gray value in the color, from 0 to $100 \%$. Reducing the saturation value toward zero to include gray and that will turn out towards faded outcome. In situations, the saturation is also expressed through $0-1$, while 0 represents true gray. The 1 is a true primary color.

Value the brightness known as intensity of the color is much related to saturation ranges from $0-100 \%$. The 0 belongs to full black, and 100 belong to white.

The graph or a plot of the distribution of the pixel intensities in an image is known as a histogram. A histogram can be calculated both for the grayscale image as well as for the color image also. The first case has only a single channel so a single histogram can be derived. In the second case can derive three histograms because there 
are three channels, calculating the histogram of an image is very useful as it gives an intuition regarding some properties of the image like tonal range, the contrast and the brightness. For our CBIR the hue scale is separated into 8 groups, saturation is separated into 2 groups, and intensity degree is separated into 2 groups.

$$
\begin{gathered}
R \frac{R}{255} \\
G \frac{G}{255} \\
B \frac{B}{255} \\
C_{\text {max }}=\max (R, G, B) \\
C_{\min }=\min (R, G, B) \\
\nabla=C_{\max }-C_{\min }
\end{gathered}
$$

Hue calculation: $H=60^{\circ} X\left(\frac{G-B}{\Lambda}\right) \bmod 6, C_{\max =R}$

$$
\begin{gathered}
60^{\circ} X\left(\frac{B-R}{\Delta}+2, C_{\max =R}\right. \\
60^{\circ} X\left(\frac{R-G}{\Delta}+2, C_{\max =B}\right. \\
\text { Saturation calculation : } S=\frac{\Delta^{0, C_{\max =0}}}{C_{\max }}, C_{\max }=0 \\
\text { Value calculation } \quad V=C_{\max }
\end{gathered}
$$

By combining all these group, in total 32 values correspond to 64-component of HSV color histogram is extracted. The three scales having a different number of groups because among the three hues is considered as the most important one following intensity in number two and finally the saturation. The individual histogram module is simplified as each pixel having the consequent color arrangement value. It is well understood that the better way of indexing of the histograms will lead to the increased performance of a CBIR to a great extent.

\subsection{Shape Descriptors}

In CBIR Shape features are always considered as principal low-level feature values of an image. There are usually two kinds of shape representation methods in the CBIR method. One is region-based and another one is contour-based. Both methods consist of structural and Global features. The Shape emission descriptors are mere numbers that are extracted from the two-dimensional shape of an image. In a few occurrences, the set of information is sufficient that the original shape can be reproduced from the shape descriptors. In some situations, the separation of shape descriptors particularly applied in convenient applications only. Shape descriptors $\underline{\underline{9}}$ are split into two categories as boundary-based normally known as contour-based and the next one is the region-based methods. It is noticeably. This classification makes us understand whether the shape feature values are retrieved from the contour of the image or considering the whole shape region of the image. Again, these two classes can be subdivided into structural descriptors (local) and global descriptors. It is by means of whether the full image shape is represented or only a part of image segments or sections are represented. Global descriptors categorize the shape description methods into spatial by considering the direct measurements of the shape and transform domain techniques, Applying a transformation to the image.

The various types of shape descriptors are listed as follows.

a. Area Based Shape Descriptors.

(i). Geometric Moments and Moment Invariants.

(ii). Shape Orientation.

(iii). Shape Elongation (Extensions).

(iv). Shape Circularity.

(v). Circularity Measures.

b. Boundary Based Shape Descriptors. $\underline{10}$

(i). Line Moments.

(ii). Boundary based shape orientation.

(iii). Convexity Measure.

c. Multi-component Shape.

\subsection{Canny Edge}

In the field of image mining, one of the hot topics to extract the features of any image is the Canny Edge Detection algorithm which performs the operation of finding the edges lie in an image. This algorithm performs multi-stage operations to achieve the edges. The edge discovery is at risk of noise in the input image. 


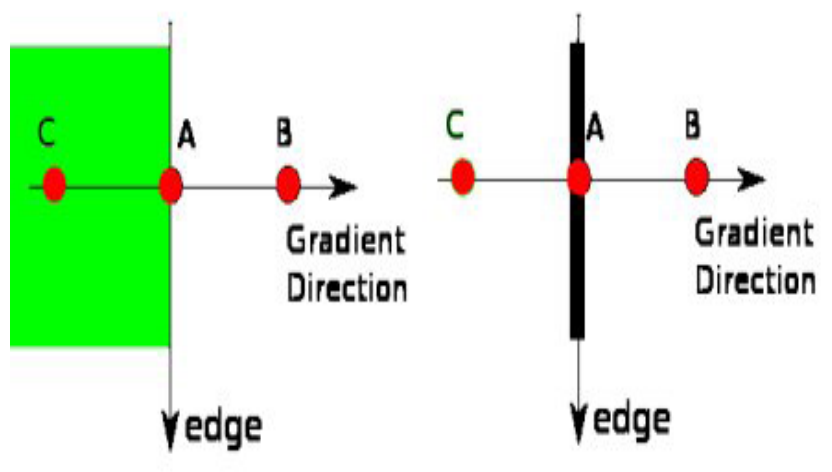

Figure 2. Canny edge extraction.

The initial operation is to take away the noise in the image by utilizing the Gaussian filter with respect to $5 \times 5$. The next process is to perform the Sobel kernel operation to acquire the first derivative of horizontal direction (Gx) and vertical direction (Gy) through filtering the image. By applying the following formula to the produced images the edge gradient and the direction for each pixel are located as shown in Figure 2.

Edge Gradient $\mathrm{G}=\sqrt{G \frac{2}{x}}+G \frac{2}{y}$

Angle $\theta=\tan ^{-1} \frac{G_{y}}{G_{x}}$

Always the Gradient direction is vertical to edges. The edge is smoothed to only one angle out of the four angles which lie in the direction towards vertical or horizontal or two crosswise directions.

When a gathering of the gradient magnitude value and direction is carried out the surplus pixels those do not comprise any contribution to finding the edges are thrown away through the complete scanning of the image. To perform this process each pixel present in the image is verified whether it is a local maximum towards its neighborhood in the direction of the gradient. The output of the process will be a thin-edged binary image. To perform the thresholding if the value of intensity gradient of edges is more than maximum threshold value they should be considered as sure edges, and which are lower than the minimum threshold value is to be considered as sure non-edges. The pixel value falls between the min and max threshold values are considered as either edge or non-edge by considering the connectivity of the pixel while forming the image. If they have a connection to sure-edge pixels they should be considered as a part of edges. If it is not, then those pixels are rejected for consideration. The Canny algorithm is an optimal edge detecting algorithm that gets a grayscale image as input to produces the output as an image screening the discontinuity of pixels with their identified intensity. It executes its operations through several levels of processing. The primary step to an input image is the performance of smoothening through Gaussian convolution. As the next step with the smoothened image, the $2 \mathrm{D}$ first-order derivative is performed. This highlights the edges of the image. Then the algorithm applies the value zero to all the pixels in which edge points are not at the top of the edges. Through this operation, it can produce a brighter line as the output image. This process is called as non-maximal suppression. The efficiency achieved by a canny edge detector by executing the three operations in an efficient way.

The first one is at the smoothening operation through the width of a Gaussian kernel. The second one is through the maximum threshold and the minimum threshold always determined by the user. The sensitivity towards the unwanted pixels will be reduced through the increased value of the width of the Gaussian kernel which can lead to the loss of some greater details of an image. One setback of a canny edge operator is in situations where three ridges meet in the slope extent of the image. To eradicate this situation the user has to consider the two

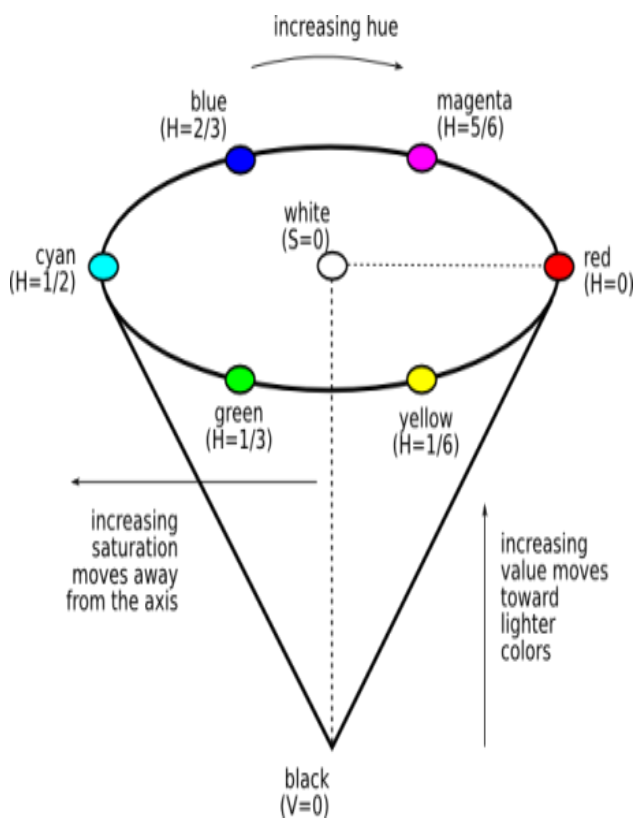

Figure 3. SVM separating hyperplane 
Table 1. Description of Kernel functions

\begin{tabular}{|c|c|c|c|}
\hline Sl.No & Kernel & Formula & Description \\
\hline 1. & $\begin{array}{l}\text { Polynomial } \\
\text { kernel }\end{array}$ & $k\left(x_{i}, x_{j}\right)=\left(x_{i} \cdot x_{j}+1\right)^{d}$ & $\begin{array}{l}\text { It is popular in image } \\
\text { processing where } d \text { is the } \\
\text { degree of the polynomial }\end{array}$ \\
\hline 2. & Gaussian kernel & $k(x, y)=\exp \left(-\frac{\|x-y\|^{2}}{2 \sigma^{2}}\right)$ & $\begin{array}{l}\text { It is a general-purpose kernel; } \\
\text { used when there is no prior } \\
\text { knowledge about the data }\end{array}$ \\
\hline 3. & $\begin{array}{l}\text { Gaussian radial } \\
\text { basis function } \\
(\mathrm{RBF})\end{array}$ & $\begin{array}{l}k\left(x_{i}, x_{j}\right)=\exp \left(-\gamma\left\|x_{i}-y_{j}\right\|^{2}\right) \\
\text { for } \gamma>0 \\
\text { at times the parameter value may be } \\
\gamma=1 / 2 \sigma^{2}\end{array}$ & $\begin{array}{l}\text { It is a general-purpose kernel; } \\
\text { used when there is no prior } \\
\text { knowledge about the data }\end{array}$ \\
\hline 4. & $\begin{array}{l}\text { Laplace RBF } \\
\text { kernel }\end{array}$ & $k(x, y)=\left(-\frac{\|x-y\|}{\sigma}\right)$ & $\begin{array}{l}\text { It is general-purpose kernel; } \\
\text { used when there is no prior } \\
\text { knowledge about the data }\end{array}$ \\
\hline 5. & $\begin{array}{l}\text { Hyperbolic } \\
\text { tangent kernel }\end{array}$ & $\begin{array}{l}k\left(x_{i}, x_{j}=\tanh \left(k x_{i}, x_{j}+c\right)\right. \\
\text { not every } \mathrm{k}>0 \text { and } \mathrm{c}<0\end{array}$ & $\begin{array}{l}\text { We can use it in neural } \\
\text { networks }\end{array}$ \\
\hline 6. & Sigmoid kernel & $k(x, y)=\tanh \left(\alpha x^{T} y+c\right)$ & $\begin{array}{l}\text { We can use it as the proxy for } \\
\text { neural networks }\end{array}$ \\
\hline 7. & $\begin{array}{l}\text { Bessel function } \\
\text { of the first kind } \\
\text { Kernel }\end{array}$ & $\begin{array}{l}k(x, y)=\frac{J_{v+1}(\sigma\|x-y\|}{\|x-y\|^{-n(v+1)}} \\
j \text { is the Bessel function of first kind }\end{array}$ & $\begin{array}{l}\text { We can use it to remove the } \\
\text { cross term in mathematical } \\
\text { functions }\end{array}$ \\
\hline 8. & $\begin{array}{l}\text { ANOVA radial } \\
\text { basis kernel }\end{array}$ & $k(x, y)=\sum_{k=1}^{n} \exp \left(-\sigma\left(x^{k}-y^{k}\right)^{2}\right)^{d}$ & $\begin{array}{l}\text { can use it in regression } \\
\text { problems }\end{array}$ \\
\hline 9. & $\begin{array}{l}\text { Linear splines } \\
\text { kernel in } \\
\text { one-dimension }\end{array}$ & $k(x, y)=1+x y+\min (x, y)-\frac{x+y}{2} \min (x, y)^{2}+\frac{1}{3} \min (x, y)^{3}$ & $\begin{array}{l}\text { It is useful when dealing with } \\
\text { large sparse data vectors. } \\
\text { It is often used in text } \\
\text { categorization. The splines } \\
\text { kernel also performs well in } \\
\text { regression problems }\end{array}$ \\
\hline
\end{tabular}

ridges as a single line sector and the third ridge as a line that coming towards the edge.

\section{Distance Metrics}

Euclidian distance $\underline{\underline{11}}$ is the popular and common distance metric which is at a higher priority to find the distance among the two points. The distance is calculated through the root square difference involving coordinates match up with two objects. In this metric Pythagorean Theorem principle is used as the primary phenomena here. There are several advantages when applying these measures are 1) Relative insensitivity to a small deviation of the CBIR system 2) very much simple to compute 3) The embedding of Euclidian distance shows increased efficiency in most of the dominant image identification performance.

$$
d j=\sqrt{\sum_{i-1}^{m}\left(q_{i}-p_{i}\right)^{2}}
$$

\subsection{SVM}

Support vector machines (SVM) perform classification $\underline{12}, \underline{13}$ by the construction of an $\mathrm{N}$-dimensional hyperplane 


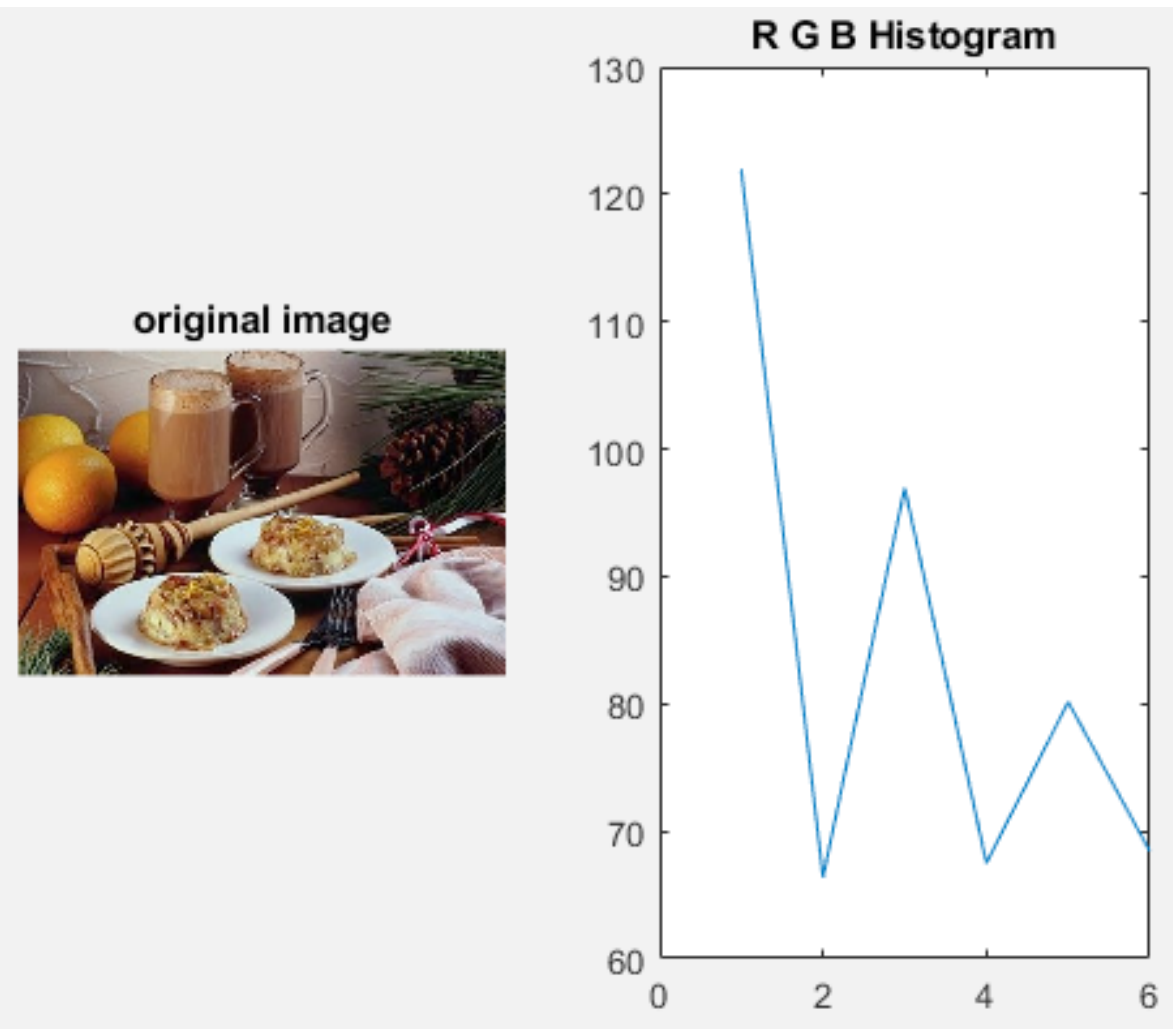

Figure 4. R G B histogram.
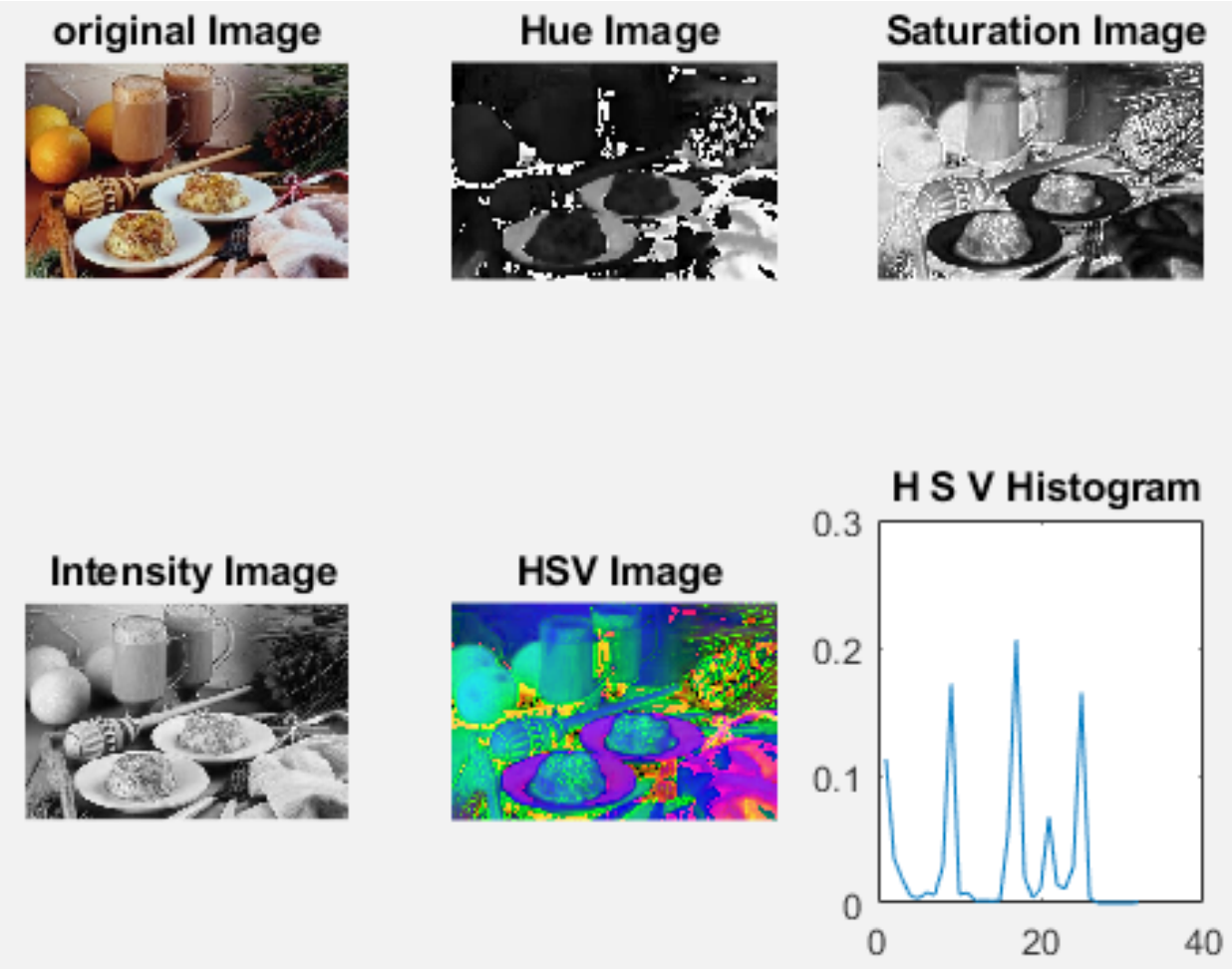

Figure 5. H S V histogram. 

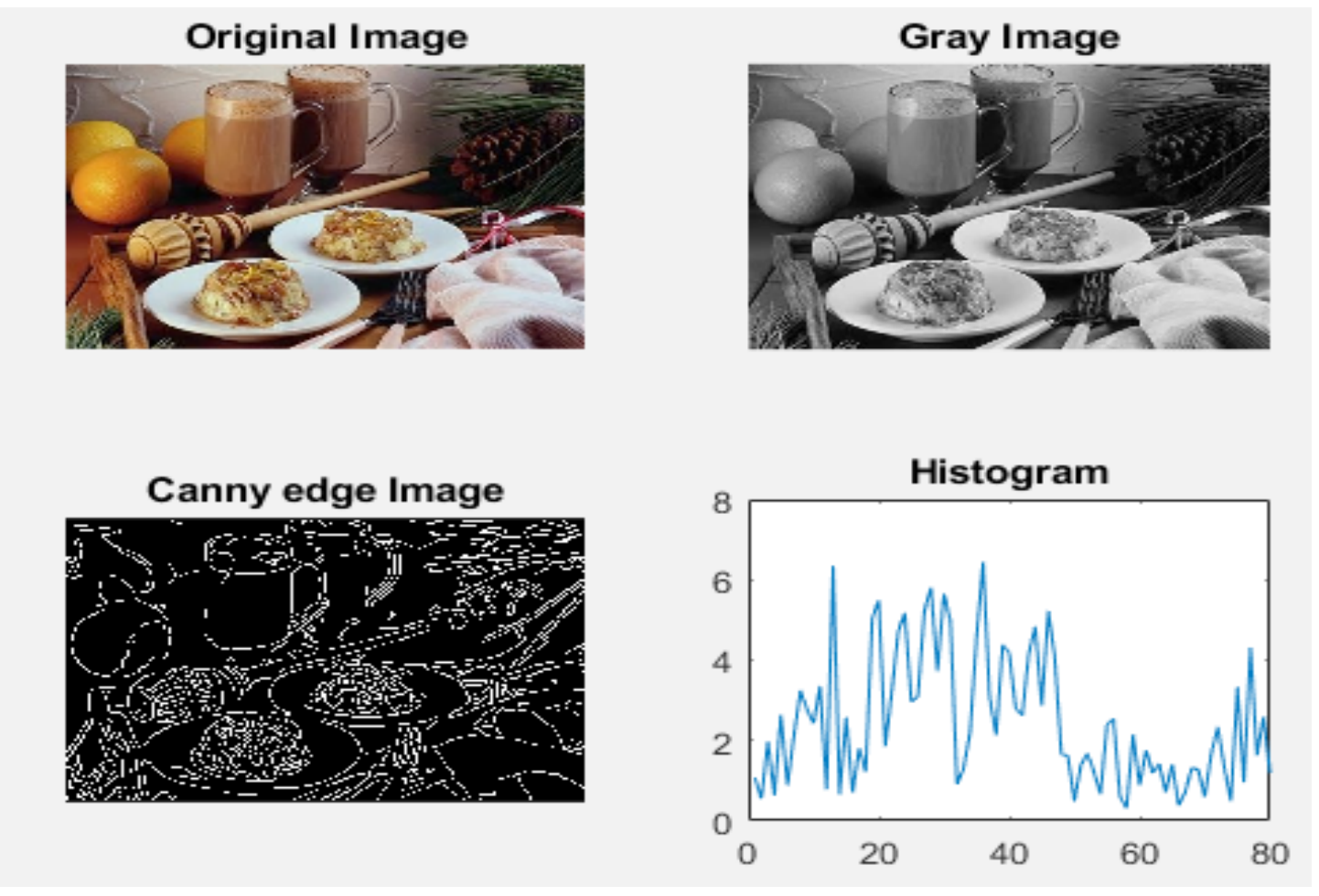

Figure 6. Canny edge histogram.

which optimally divides the data into two categories. SVM models are strongly associated with neural networks. The SVM representation by means of a sigmoid kernel function is identical to a neural network approach known as the bi-layer feed-forward model. Through kernel function, SVM's have substitutive learning processes to polynomial kernel, radial basis function and multi-layer perceptron approach. The weights of the network are set up by solving a quadratic encoding problem using the linear constraint, rather than by solving a non-convex, unconstrained minimization problem through the standard neural network learning process. The purpose of SVM modeling is to discover the best possible hyperplane that separates clusters of the vector in such a way that cases with one grouping of the target objects grouped to one side of the plane and remaining grouping are on the next side of the plane. It is given in Figure 3. The vectors near the hyperplane are the support vectors. SVM learning is a valuable statistic approach towards machine learning procedure that has been effectively practicing in pattern identification. If the data are linearly non-separable but nonlinearly separable, the nonlinear support vector classifier will be applied. The basic idea is to renovate input vectors into a high-dimensional characteristic vector by means of a nonlinear renovation, and then to do a linear separation in feature space. To assemble a nonlinear SVM, the internal product is transformed through kernel function. $\underline{14}$

The SVM has two layers. At some stage in the learning phase, the first layer chooses the basis, (as well as the number) from the known set of bases determined with the help of the kernel then the second layer builds a linear function in this space. This is entirely correspondent to build the best possible hyperplane in the subsequent feature space. The SVM algorithm can create a range of learning approaches by applying suitable kernel $1 \underline{15}$ functions. Table 1 shows a few kernel functions and their descriptions.

\section{Results and Discussion}

In the proposed model the well-know $\underline{16}$ dataset is considered for performing the experiments. The dataset consists of ten categories of different images. 100 images per category evolve in a total of 1000 image collection. To produce a reliable fast CBIR methodology the color and shape characteristics of an image are combined 

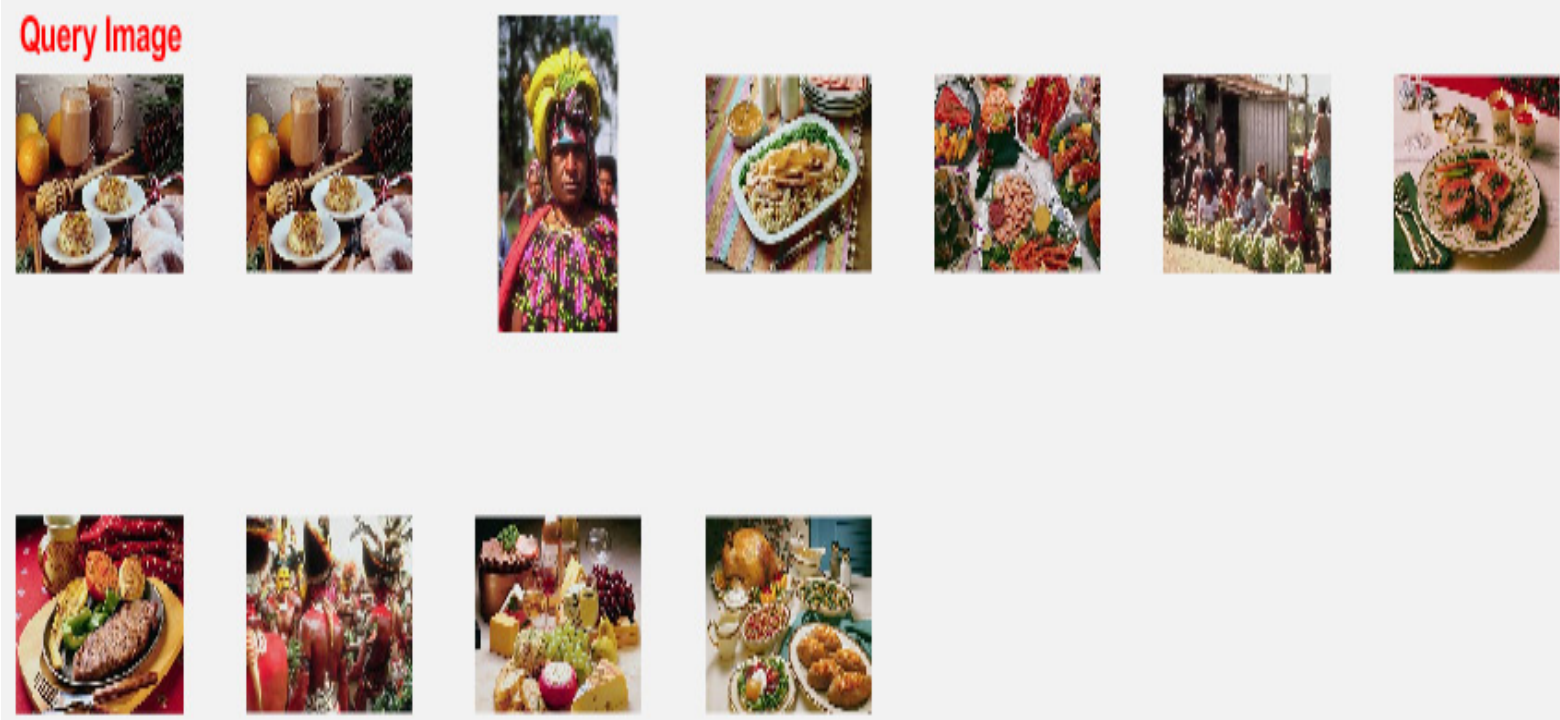

Figure 7. Image retrieval for categorization.
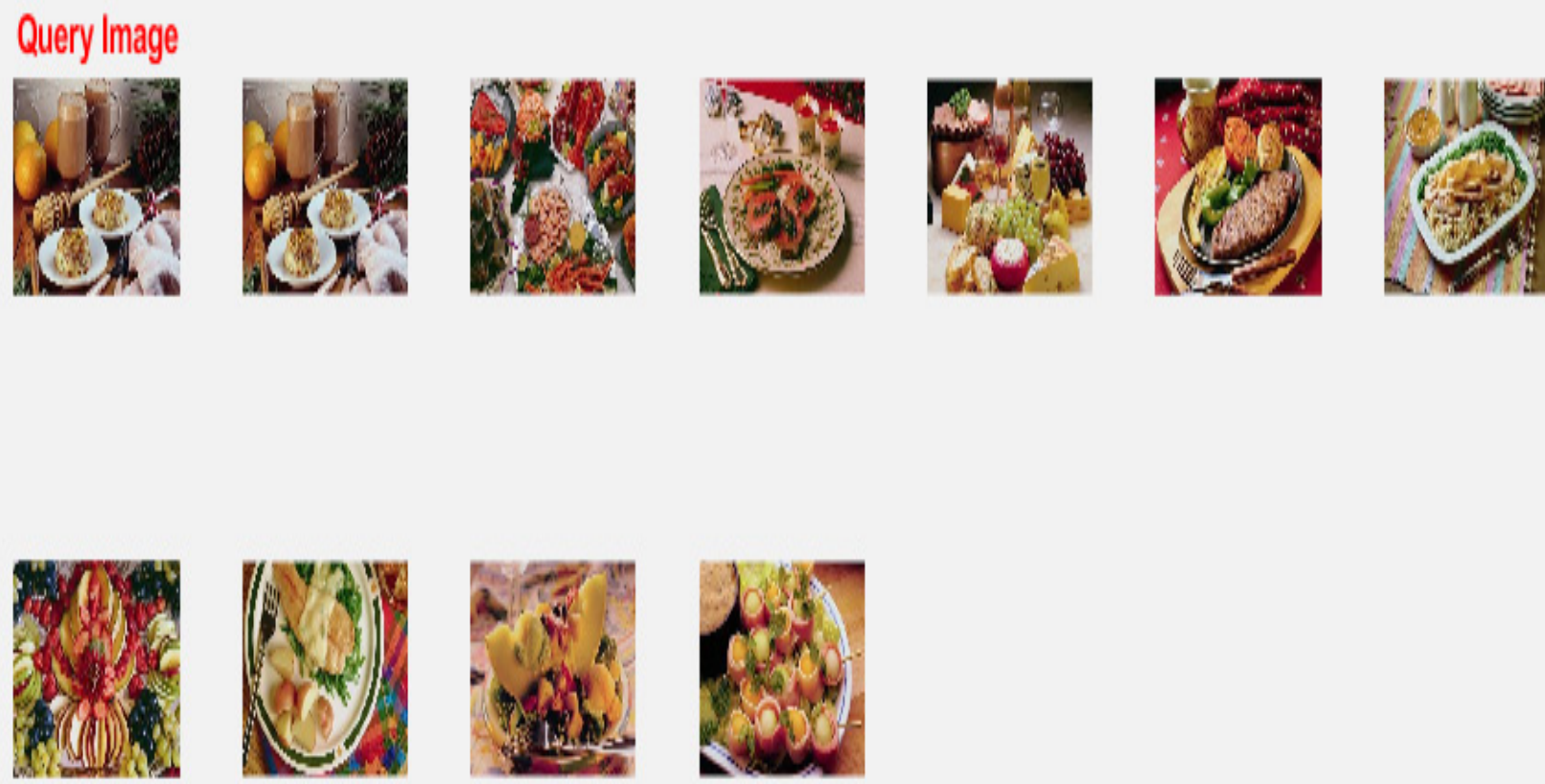

Figure 8. Classification based image retrieval.

to produce the HFDC. $\frac{17}{7}$ The proposed method was designed through Matlab. The RGB histogram and HSV histogram features are extracted to represent the color model of the images. To compute the RGB histogram the mean value for each of the prime colors and the standard deviation of the prime color in total 6 features are extracted. Figure 4 shows the RGB histogram computed values.

The HSV extracts 8 bins for hue and 2 bins for saturation and 2 bins for intensity value. A total of 32 HSV features are extracted. The following Figure 5 shows the HSV computed features. 


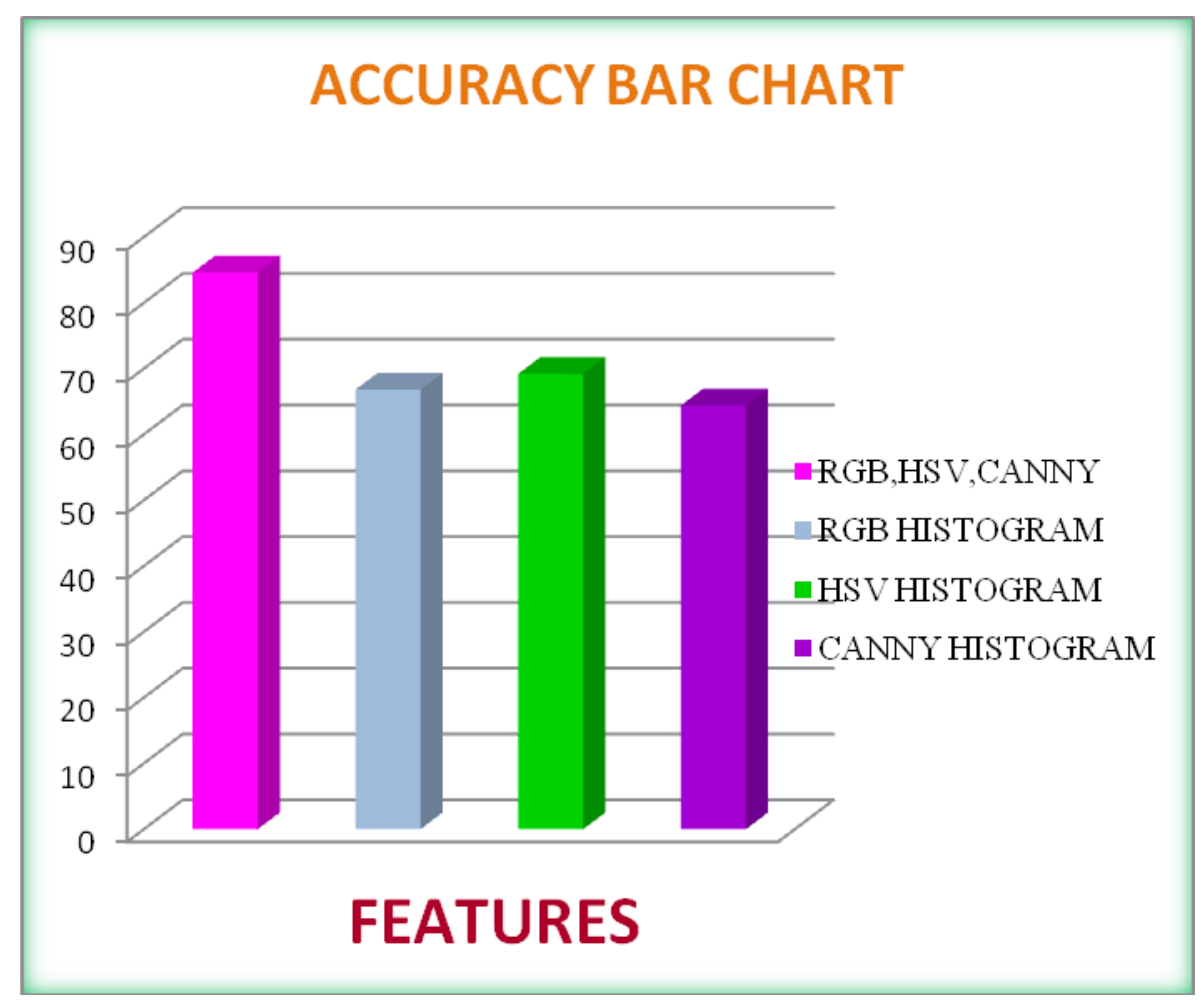

Figure 9. Overall performance of SVM-RBF kernel.

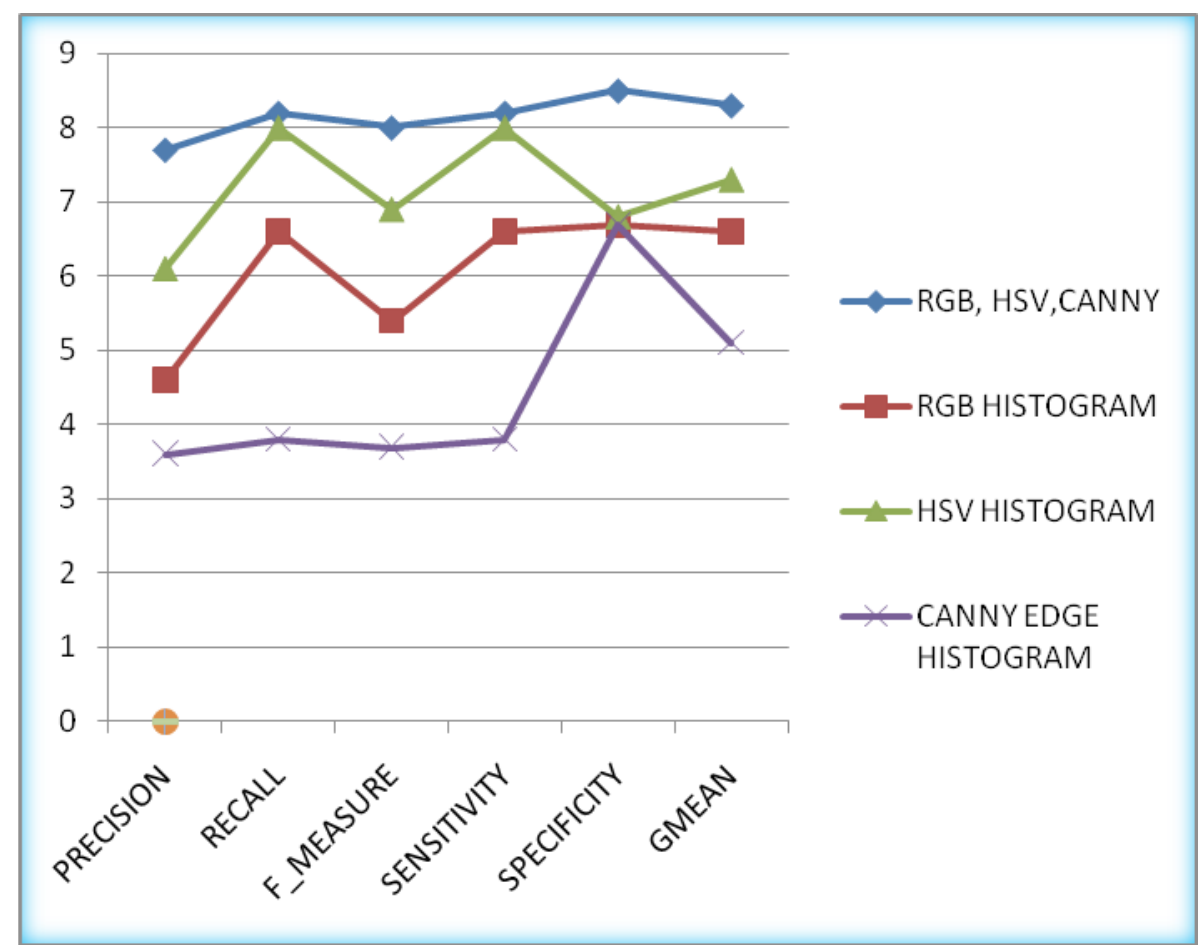

Figure 10. Comparison of classification performance. 
To identify the edges of the images the well known and widely used canny edge Histogram is computed. Through a canny edge histogram which is shown in Figure 6. Consist of 80 features is extracted. After extracting the features, the RGB HSV and canny histogram features are stored as a single feature vector for each image present in the corpus. Totally 118 appropriate image feature integration $\frac{18}{}$ per image is used to represent each image present in the dataset. The comparison among the query image with the database image is performed using the Euclidean in our proposed model.

Hear the proposed method tries to get back ten images most similar to the query image from the overall image corpus. The query image features are used as an input feature vector to get back the most identical images through SVM-RBF kernel to determine the corresponding category of the query image. The retrieved images are shown in Figure 7. After determining the relevant group of query image the proposed method attempts to retrieve ten most similar images corresponding to that particular category.

The 10 most significant images retrieved from the resultant category of the images by the SVM are listed in Figure 8. In our experiment, the RBF kernel function applied to train the SVM. Prior to beginning the testing process, the dataset is divided into $50 \%$ as a training set and $50 \%$ as the testing set to train the SVM.

Figure 9 express the accuracy retrieved by the SVM through RGB, HSV and canny edge features individually as well as using the HFDC. Figure 10 illustrate the overall performance evaluation of the classification by SVM-RBF kernel.

The evaluations are carried out through determining confusion metrics. It clearly displays that the HFDC of color and shape features produce better performance by achieving good accuracy compared with other extracted feathers utilized for classification atomically.

\section{Conclusion}

The traditional supervised learning methods always tend to produce the amost significant results in the field of CBIR. The hurried raise in the enormous use of visual content influenced to develop many retrieval systems. This development made happening by retrieving the visual content through various image features as a vital source of descriptors to represent the visual content. Developing new algorithms to combine various image characteristics of visual content to produce hybrid feature vectors to match images with a query from users can be very much effective to produce better and accurate results. Giving focus on this issue the proposed method performs image classification using CBIR to satisfy the user requirement. Here the proposed method makes use of color and shape characteristics of the image as features to execute the CBIR based image classification. These features predominance are evaluated and compared with each other and as well as with HFDC through classification. The proposed method yields an improved accuracy of $84.60 \%$ while combining the RGB histogram, HSV histogram and canny edge histogram features to produce the HFDC as a visual content descriptor. Further the same can also be extended to achieve the clustering results through various clustering algorithms like DBSCAN and K-MEANS clustering algorithms.

\section{References}

1. Haji MS, Alkawaz MH, Rehman A, Saba T. Content-based image retrieval: a deep look at features prospectus. Int J Comput Vis Robot. 2019;9(1):14-38.

2. Shriram K, Arumugam B, Priyadarsini PLK. An intelligent system of content-based image retrieval for crime investigation. Int J Adv Intell Paradig. 2015;7(3):264-79.

3. Muller H, Michoux N, Bandon D, Geissbuhler A. A review of content-based image retrieval systems in medical applications - clinical benefits and future directions. Int J Med Inf. 2004;73(1):1-23.

4. Goksu O, Aptoula E. Content based image retrieval of remote sensing images based on deep features. 26th Signal Processing and Communications Applications Conference (SIU). 2018. vol 10 (3). P. 664-74.

5. Zhang J, Zhang J, Dai T, He Z. Exploring weighted dual graph regularized non-negative matrix tri-factorization based collaborative filtering framework for multi-label annotation of remote sensing images. Remote Sens. 2019;11(5):493.

6. Cao J, Wang M, Shi H, Hu G, Tia Y. A new approach for large-scale scene image retrieval based on improved parallel-means algorithm in MapReduce environment. Math Probl Eng. 2016:1-17.[AQ3]

7. Kumari B. CBIR systems: results of feature extraction with color feature comparison with standard database. Int J Innov Res Comput Commun Eng. 2017;5(11):2576-81.

8. Boulemden A, Tlili Y, Jalab HA. Content-based image retrieval with pachinko allocation model and a combination 
of colour, texture and text features. Int J Comput Vis Robot. 2018;8(2):122-39.

9. Rostami R, Bashiri FS, Rostami B, Yu Z. A survey on datadriven $3 \mathrm{D}$ shape descriptors. computer graphics forum. 2018;38(1):356-93.[AQ4]

10. Content-based image retrieval using new color histogram. [cited 2004 Dec]. Available from: https://www.researchgate. net/publication/4148180_Content-based_image_retrieval_ using_new_color_histogram.

11. Mistry Y, Ingole DT, Ingole MD. Content based image retrieval using hybrid features and various distance metric. J Electr Syst Inf Technol. 2018;5(3):874-88.

12. Wei Khor J, Jean N, Luxenberg ES, Ermonc S, Tang SKY. Using machine learning to discover shape descriptors for predicting emulsion stability in a microfluidic channel. Soft Matter Emerg Investig. 2019;6:1079-86.

13. Ansari MA, Kurchaniya D, Dixit M, Kumar Johari P. An effective approach to an image retrieval using SVM classifier. Int J Comput Sci Eng. 2018;5(6):62-72.
14. Prakash Singh V, Srivastava R. Effective image retrieval based on hybrid features with weighted similarity measure and query image classification. Int J Comput Vis Robot (IJCVR). 2018;8(2):98-121.

15. Analysis of SVM kernels for content based image retrieval system. [cited 2017 Aug 01]. Available from: https:// ieeexplore.ieee.org/document/8389676.

16. Ahmed KT, Ummesafi S, Iqbal A. Content based image retrieval using image features information fusion. Inf Fusion. 2019;51:76-99.

17. Unar S, Wanga X, Wang C, Wanga Y. A decisive content based image retrieval approach for feature fusion in visual and textual images. Knowl Based Syst. 2019;179:8-20.

18. Jyothi B, Madhavee Latha Y, Krishna Mohan PG, Reddy VSK. Integrated multiple features for tumor image retrieval using classifier and feedback methods. Procedia Comput Sci. 2016;85:141-8. 